\title{
Power-induced lasing state switching and bistability in a two-state quantum dot laser subject to optical injection
}

\author{
Zaifu Jiang ${ }^{1,2}$, Zhengmao Wu $^{1 *}$, Elumalai Jayaprasath ${ }^{1}$, Wenyan Yang ${ }^{1,3}$, \\ ChunXia Hu ${ }^{1,4}$, Bing Cui ${ }^{1}$, Guangqiong XiA ${ }^{1 *}$
}

${ }^{1}$ School of Physical Science and Technology, Southwest University, Chongqing 400715, China

${ }^{2}$ School of Mathematics and Physics, Jingchu University of Technology, Hubei 448000, China

${ }^{3}$ School of Physics, Chongqing University of Science and Technology, Chongqing 401331, China

${ }^{4}$ College of Mobile Telecommunications, Chongqing University of Posts and Telecom, Chongqing 401520, China

${ }^{*}$ Corresponding authors: Z.M. Wu (zmwu@swu.edu.cn) and G.Q. Xia (gqxia@swu.edu.cn)

\begin{abstract}
We theoretically investigate power-induced lasing state switching and bistability in a two-state quantum dot laser subject to optical injection. The simulated results show that, for a free-running two-state quantum dot laser operating at the ground state under low current, a power-induced lasing state switching between the ground state and the excited state can be achieved through introducing optical injection with a frequency $\left(w_{\text {inj }}\right)$ close to the lasing frequency of excited state $\left(w_{\mathrm{ES}}\right)$. The injection power required for the state switching depends on the scanning route of injection power, i.e. there may exist state bistability for the injection power within a certain region. For forward scanning injection power, with the increase of frequency detuning $\left(\Delta \Omega=w_{\text {inj }}-w_{\mathrm{ES}}\right)$, the injection power required for the state switching shows a decreasing trend accompanied by slight fluctuations. However, for backward scanning injection power, the injection power required for the state switching exhibits obvious fluctuations with the increase of $\Delta \Omega$. The width of the hysteresis loop fluctuates with $\Delta \Omega$, and the fluctuation amplitude is increased with the increase of the injection current. Additionally, the influences of the inhomogeneous broadening factor and the electron escape rate on the bistability performances are analyzed.
\end{abstract}

Keywords: two-state quantum dot lasers, lasing state switching, bistability, optical injection.

\section{Introduction}

Optical switching and bistability have attracted much attention due to their wide application prospects in optical memory, optical logic-gate, optical information processing, and so on [1-7]. Previous studies have demonstrated that semiconductor lasers (SLs) can exhibit diverse switching and bistability behaviors under different external pertur- 
bations [ $\underline{8}-\underline{15}]$. For instance, BRANDONISIO et al. have exhibited the mode switching based on a two-mode SL under current modulation, and found that the switching time could be less than $2 \mathrm{~ns}$ under appropriate modulation parameters []]. Li et al. have discovered the recurrent switching phenomenon between two modes of semiconductor ring laser under counter-directional mutual feedback [9]. BLIN et al. have reported the dynamical state bistability in a distributed-feedback (DFB) semiconductor laser subject to optical injection [10]. Besides, the polarization switching (PS) and bistability (PB) based on vertical-cavity surface emitting laser (VCSEL) have also been extensively studied. For example, ZHANG et al. have demonstrated the PS in a VCSEL subject to orthogonally-polarized optical injection [11]. HURTADO et al. have experimentally studied the PB in a VCSEL under orthogonally-polarized optical injection, and observed three different shapes of $\mathrm{PB}$ [12]. In addition, our group has experimentally studied the effect of the polarization angle on the PB characteristics of a VCSEL subject to variable polarization optical injection, and found that the hysteresis width decreases with the increase of polarization angle [13] .

Among different types of SLs, a self-organized SL with a quantum dot (QD) structure has attracted much interest [ $\underline{16}-\underline{25}]$ due to such unique properties as low relative intensity noise [26], weak temperature dependence [27], low transparency current density [료], as well as reduced sensitivity for optical feedback [29]. If the wavelength selection technology is not used in the manufacturing process, a free-running QD laser may lase in a ground state (GS), an excited state (ES), or a GS and ES coexistence state under different currents [30]. Correspondingly, such QD lasers are named as two-state QD lasers. For a two-state QD laser, with the gradual increase of the bias current from zero, the recombination of the electrons and holes corresponding to the GS will reach the first threshold and then results in the GS emission. Continuously increasing the bias current, the population of the ES increases. Once the current exceeds the secondary threshold, the two-state QD laser simultaneously lases in both the GS and the ES. Further, the two-state QD laser may lase in the ES only if the bias current is high enough $[\underline{30}, \underline{31}]$. According to relevant experimental reports, for a self-organized InAs two -state QD laser, the lasing wavelengths of the GS and ES are approximately 1300 and $1220 \mathrm{~nm}$, respectively [31]. Such a large wavelength difference between the two lasing states makes the two-state QD laser be a good candidate for optical switching device. Recently, the lasing state switching and bistability based on the two-state QD lasers under optical feedback and optical injection have been investigated. For example, MEINECKE et al. have investigated the bistability characteristics of a two-state QD laser subjected to an optical injection and found that the size of the bistability strongly depends upon the pump current and the amplitude-phase coupling [32]. VIRTE et al. have demonstrated theoretically and experimentally the state competition and switching between the GS and ES states for a two-state QD laser subject to optical feedback [ $\underline{33}, \underline{34}]$, and the successive switching repetition between the ES and GS can be achieved by changing the external cavity feedback time. TYKALEwICZ et al. have studied the lasing state switching and bistability characteristics of a two-state QD laser under optical injection $[\underline{31}, \underline{35}, \underline{36}]$, and the results show that, for a free-running two-state QD laser operating 
at ES, the lasing state switching with fast state switching time and high state suppression rate, and the lasing bistability with large hysteresis loop width can be achieved through introducing an injection light with a frequency close to the GS lasing frequency.

As mentioned above, the current of a free-running two-state QD laser at the ES lasing is much higher than that of the two-state QD laser at the GS lasing. Therefore, the lasing state switching and bistability of a free-running two-state QD laser operating at the GS lasing may be more promising for some practical applications. Based on this consideration, in this work, for a free-running two-state QD laser operating at the GS, after introducing an optical injection with a frequency near the ES lasing frequency, we investigate the power-induced lasing state switching and bistability through forward and backward scanning the injection power. For the two-state QD laser biased at different currents, the variations of the injection powers required for the state switching and the hysteresis loop width with the frequency detuning are discussed. Moreover, the influences of the inhomogeneous broadening factor and the electron escape rate on the bistability characteristics are analyzed.

\section{Theoretical model}

Figure 1 shows the energy level structure and the carrier dynamics of a two-state QD laser. The energy levels are discrete due to the three-dimensional restriction of the QD structure [30]. Here, all QDs are assumed to possess the same size, and therefore only one QD ensemble is considered in the active region [30]. Via the electrodes, the carriers are directly injected into the wetting layer (WL) plane, and then some carriers with the rates of $B_{\mathrm{e}, \mathrm{h}}^{\mathrm{WL}}\left(B_{\mathrm{e}, \mathrm{h}}\right)$ are captured from the WL (ES) to the ES (GS) by Auger and phonons assisted scattering processes [37, 38]. Meanwhile, some carriers will escape from ES (GS) to WL (ES) due to the thermal excitation $[\underline{30}, \underline{38}]$, and $C_{\mathrm{e}, \mathrm{h}}^{\mathrm{WL}}\left(C_{\mathrm{e}, \mathrm{h}}\right)$

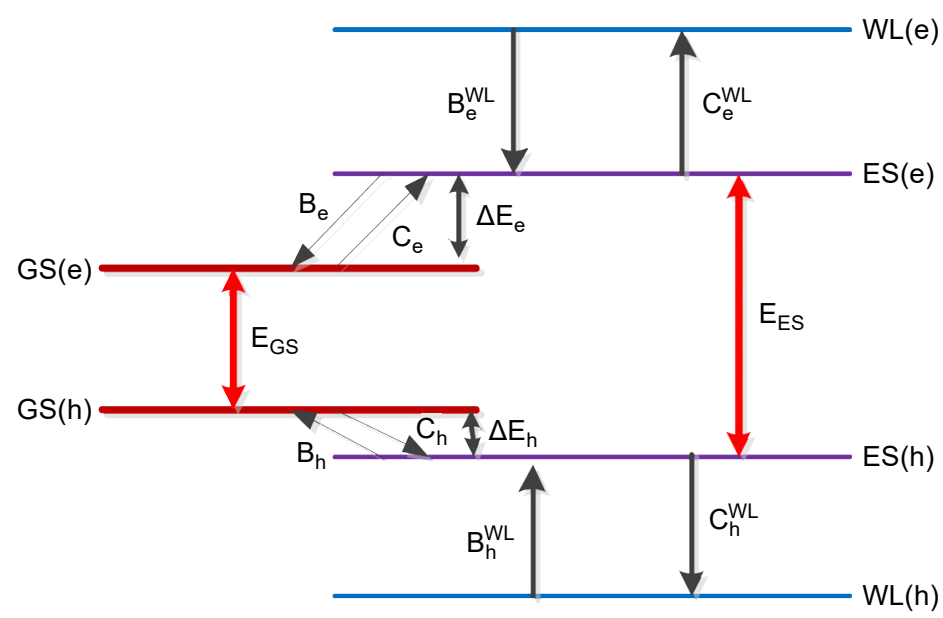

Fig. 1. Energy level structure and carrier dynamics of a two-state QD laser. WL, ES, GS represent the wetting layer, the excited state and the ground state, e and h represent the electron and the hole, $\mathrm{B}$ and $\mathrm{C}$ represent the capture and escape rates. 
denote the escape rates. Stimulated radiation can occur in the ES transition and the GS transition, and the two transition energies are $E_{\mathrm{ES}}$ and $E_{\mathrm{GS}}$, respectively. In addition, $\Delta E_{\mathrm{e}}$ and $\Delta E_{\mathrm{h}}$ represent energy level spacing of the electrons and holes between ES and GS. Combining Refs. [ $[\underline{30}, \underline{33}]$, after considering the influence of the wetting layer on the carrier dynamics, the rate equations for an optical injection two-state QD laser can be described by

$$
\begin{aligned}
& \frac{\mathrm{d} I^{\mathrm{GS}}}{\mathrm{d} T}=\left[2 g_{0}^{\mathrm{GS}}\left(n_{\mathrm{e}}^{\mathrm{GS}}+n_{\mathrm{h}}^{\mathrm{GS}}-1\right)-1\right] I^{\mathrm{GS}} \\
& \frac{\mathrm{d} E^{\mathrm{ES}}}{\mathrm{d} T}=\frac{1}{2}\left[(1+i \alpha)\left(4 g_{0}^{\mathrm{ES}}\left(n_{\mathrm{e}}^{\mathrm{ES}}+n_{\mathrm{h}}^{\mathrm{ES}}-1\right)-1\right)\right. \\
& \left.+i 2 \beta g_{0}^{\mathrm{GS}}\left(n_{\mathrm{e}}^{\mathrm{GS}}+n_{\mathrm{h}}^{\mathrm{GS}}-1\right)\right] E^{\mathrm{ES}}+\varepsilon \exp (i \Delta \Omega T) \\
& \frac{\mathrm{d} n_{\mathrm{e}, \mathrm{h}}^{\mathrm{GS}}}{\mathrm{d} T}=\eta\left[2 B_{\mathrm{e}, \mathrm{h}} n_{\mathrm{e}, \mathrm{h}}^{\mathrm{ES}}\left(1-n_{\mathrm{e}, \mathrm{h}}^{\mathrm{GS}}\right)-2 C_{\mathrm{e}, \mathrm{h}} n_{\mathrm{e}, \mathrm{h}}^{\mathrm{GS}}\left(1-n_{\mathrm{e}, \mathrm{h}}^{\mathrm{ES}}\right)\right. \\
& \left.-n_{\mathrm{e}}^{\mathrm{GS}} n_{\mathrm{h}}^{\mathrm{GS}}-g_{0}^{\mathrm{GS}}\left(n_{\mathrm{e}}^{\mathrm{GS}}+n_{\mathrm{h}}^{\mathrm{GS}}-1\right) I^{\mathrm{GS}}\right] \\
& \frac{\mathrm{d} n_{\mathrm{e}, \mathrm{h}}^{\mathrm{ES}}}{\mathrm{d} T}=\eta\left[-B_{\mathrm{e}, \mathrm{h}} n_{\mathrm{e}, \mathrm{h}}^{\mathrm{ES}}\left(1-n_{\mathrm{e}, \mathrm{h}}^{\mathrm{GS}}\right)+C_{\mathrm{e}, \mathrm{h}} n_{\mathrm{e}, \mathrm{h}}^{\mathrm{GS}}\left(1-n_{\mathrm{e}, \mathrm{h}}^{\mathrm{ES}}\right)+B_{\mathrm{e}, \mathrm{h}}^{\mathrm{WL}} n_{\mathrm{e}, \mathrm{h}}^{\mathrm{WL}}\left(1-n_{\mathrm{e}, \mathrm{h}}^{\mathrm{ES}}\right)\right. \\
& \left.-C_{\mathrm{e}, \mathrm{h}}^{\mathrm{WL}} n_{\mathrm{e}, \mathrm{h}}^{\mathrm{ES}}-n_{\mathrm{e}}^{\mathrm{ES}} n_{\mathrm{h}}^{\mathrm{ES}}-g_{0}^{\mathrm{ES}}\left(n_{\mathrm{e}}^{\mathrm{ES}}+n_{\mathrm{h}}^{\mathrm{ES}}-1\right)\left|E^{\mathrm{ES}}\right|^{2}\right] \\
& \frac{\mathrm{d} n_{\mathrm{e}, \mathrm{h}}^{\mathrm{WL}}}{\mathrm{d} T}=\eta\left[J-n_{\mathrm{e}}^{\mathrm{WL}} n_{\mathrm{h}}^{\mathrm{WL}}-4 B_{\mathrm{e}, \mathrm{h}}^{\mathrm{WL}} n_{\mathrm{e}, \mathrm{h}}^{\mathrm{WL}}\left(1-n_{\mathrm{e}, \mathrm{h}}^{\mathrm{ES}}\right)+4 C_{\mathrm{e}, \mathrm{h}}^{\mathrm{WL}} n_{\mathrm{e}, \mathrm{h}}^{\mathrm{ES}}\right]
\end{aligned}
$$

where superscripts GS, ES, and WL stand for the ground state, the excited state and the wetting layer, respectively, and subscripts e and h represent the electron and hole, respectively. For this model, the rate equations are composed of eight dimensionless equations and have taken the asymmetric electron-hole dynamics into account [30]. $T=t / \tau_{\mathrm{p}}\left(\tau_{\mathrm{p}}\right.$ is the photon lifetime) denotes the normalized time, $I^{\mathrm{GS}}$ and $E^{\mathrm{ES}}$ represent the field intensity of the GS and the complex electric field of the ES, $n$ represents the occupational probabilities. The term $(1-n)$ is the Pauli-blocking factor [37]. $2 g_{0}^{\mathrm{GS}}$ and $4 g_{0}^{\mathrm{ES}}$ denote the gains of two lasing states, where the factors 2 and 4 represent energy level spin degeneracy for the GS and ES, and $g_{0}^{\mathrm{GS}}$ and $g_{0}^{\mathrm{ES}}$ are the effective gain factors scaled to the cavity losses and are identical for the GS and ES [30]. The dimensionless parameter $\eta$ represents the carrier recombination time normalized by $\tau_{\mathrm{p}}, \alpha$ is the linewidth enhancement factor (LEF), $\beta$ is the inhomogeneous broadening factor introduced in Ref. [36], $J$ is the normalized injection current. $\varepsilon$ represents the normalized injected strength, and $\Delta \Omega=w_{\text {inj }}-w_{\mathrm{ES}}$ denotes the frequency detuning between the frequency of the injection light $\left(w_{\text {inj }}\right)$ and the lasing frequency of the ES $\left(w_{\mathrm{ES}}\right)$. Equations (1)-(5) 
T a b 1 e. Parameters used in numerical simulation [33].

\begin{tabular}{lll}
\hline Symbol & Value & Meaning \\
\hline$\eta$ & 0.02 & The ratio of the photon lifetime to the carrier lifetime \\
$g_{0}^{\mathrm{GS}}\left(g_{0}^{\mathrm{ES}}\right)$ & 0.75 & GS (ES) effective gain factor scaled to the losses \\
$\alpha$ & 5 & Linewidth enhancement factor \\
$B_{\mathrm{e}}\left(B_{\mathrm{h}}\right)$ & 150 & Electron (hole) capture rate from ES to GS scaled to the carrier lifetime \\
$B_{\mathrm{e}}^{\mathrm{WL}}\left(B_{\mathrm{h}}^{\mathrm{WL}}\right)$ & 150 & Electron (hole) capture rate from WL to ES scaled to the carrier lifetime \\
$C_{\mathrm{e}}^{\mathrm{WL}}\left(C_{\mathrm{h}}^{\mathrm{WL}}\right)$ & 1 & Electronic (hole) escape rate from ES to WL scaled to the carrier lifetime \\
$C_{\mathrm{h}}$ & 150 & Hole escape rate from GS to ES scaled to the carrier lifetime \\
\hline
\end{tabular}

can be numerically solved by using the fourth-order Runge-Kutta algorithm, and the parameters used in numerical simulations are listed in the Table.

\section{Results and discussion}

In order to specify the lasing characteristics of a two-state QD laser under different currents, Fig. 2a displays the power-current $(P-I)$ curve under $C_{\mathrm{e}}=8$ and $\beta=3$. As shown in this diagram, when the current is increased to 3.5, the GS begins to lase. For the current within the range of 3.5-8.0, the free-running QD laser is operating at the GS. Once the current exceeds 8.0, the ES is also excited, and then the free-running QD laser is operating at the coexistence of the GS and ES. With the increase of the current, the output power of the ES linearly increases while the output power of the GS gradually decreases. As pointed out in Refs. $[\underline{30}, \underline{33}, \underline{36}], C_{\mathrm{e}}$ is an important parameter for the two-state QD lasers, and its value depends on the electron energy level spacing $\left(\Delta E_{\mathrm{e}}\right)$ and the operation temperature of the laser. Under the other parameters given above, the threshold currents for the GS and ES are varied with $C_{\mathrm{e}}$, which is shown in Fig. 2b. Ob-
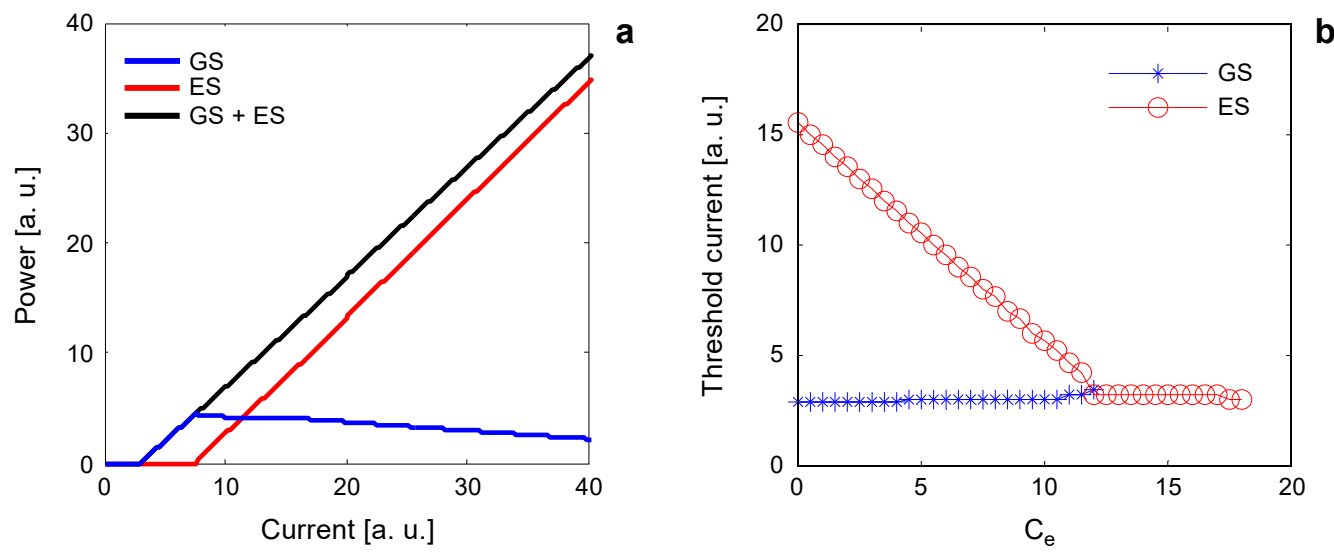

Fig. 2. Power-current $(P-I)$ curve under electron escape rate $C_{\mathrm{e}}=8$ and $\beta=3$ (a); threshold current of ES and GS versus $C_{\mathrm{e}}$ under $\beta=3$ (b). 

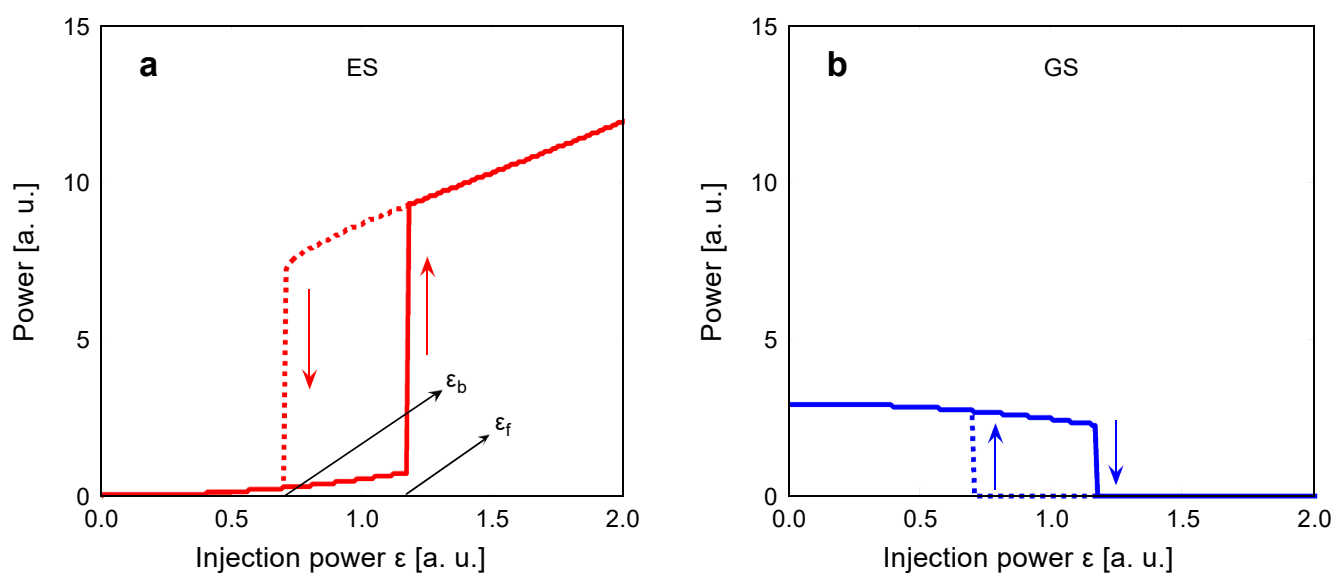

Fig. 3. Output power curves of the ES lasing (a) and the GS lasing (b) as the injected power $\varepsilon$ gradually increases (solid line) and decreases (dotted line) under $\Delta \Omega=0, J=6, C_{\mathrm{e}}=8$ and $\beta=3$.

viously, the threshold current for the GS is almost maintained at the same level for $C_{\mathrm{e}}$ varied within $(0,12)$. Once $C_{\mathrm{e}}$ is beyond 12 , the GS stops lasing. However, with the increase of $C_{\mathrm{e}}$, the threshold current for the ES firstly decreases and then also stays at the same level for the case of $C_{\mathrm{e}}>12$.

Through scanning the power of the injected light forward and backward, the power -induced state switching and bistability in an optically injected two-state QD laser can be observed. Figure 3 shows the output power of the ES and the GS as a function of the injection power under the frequency detuning $\Delta \Omega=0$, the bias current $J=6, C_{\mathrm{e}}=8$ and $\beta=3$. From this diagram, it can be seen that, during forward scanning $\varepsilon$ from 0 to 2.00 , the ES emission is stimulated and its output power gradually increases due to the introduction of optical injection, while the GS power gradually decreases due to the carrier competition mechanism between the ES and the GS [37]. Similar behavior has been experimentally observed for a two-state QD laser emitting at the ES only when the injection light frequency is closed to the GS emission frequency [36]. Once the injection power arrives at a specific value of $\varepsilon_{\mathrm{f}}=1.18$, the switching from the GS lasing to the ES lasing occurs, beyond which the ES lasing is dominant and the GS lasing is suppressed. By gradually decreasing $\varepsilon$ from 2.00 to 0 (backward scanning), the lasing state switching occurs at another specific injection power $\varepsilon_{\mathrm{b}}=0.70$, which is smaller than $\varepsilon_{\mathrm{f}}$. Therefore, the injected power required for the state switching depends on the scanned route of $\varepsilon$, which originates from different initial conditions of forward and backward scanning and then results in the emergence of a hysteresis loop. The hysteresis width is defined as $\Delta \varepsilon=\varepsilon_{\mathrm{f}}-\varepsilon_{\mathrm{b}}$, which is about 0.48 under the above given parameter values.

For an external optical injection laser, the laser bias current and the frequency detuning between the injected light and the laser are two important factors to affect the output 

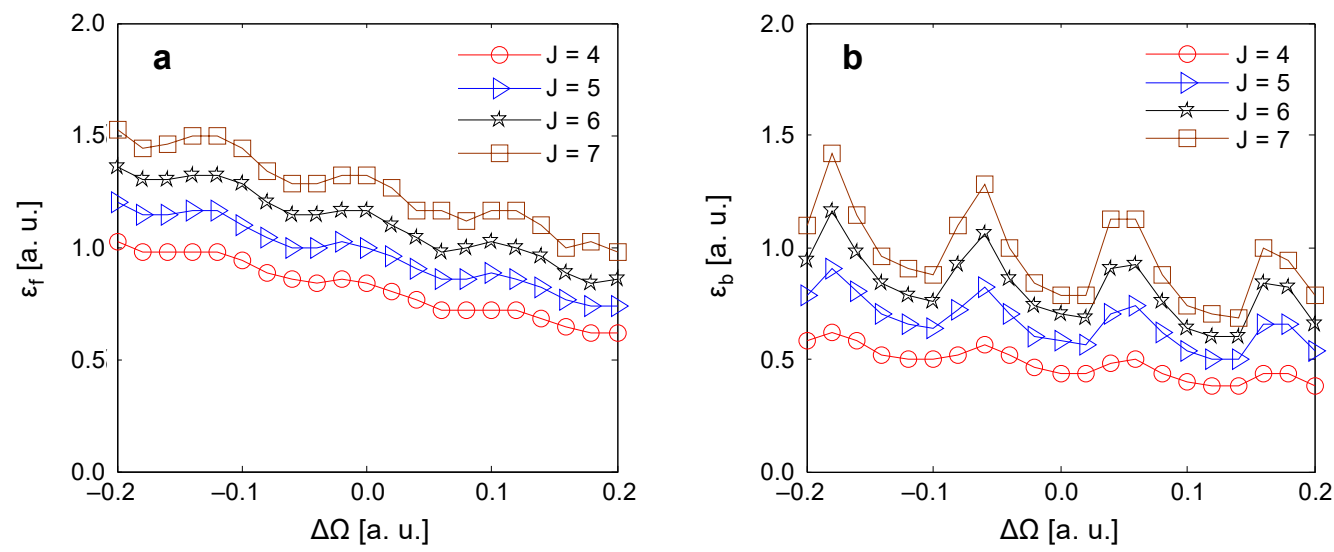

Fig. 4. Injection powers $\varepsilon_{\mathrm{f}}$ and $\varepsilon_{\mathrm{b}}$ required for the state switching versus the frequency detuning $\Delta \Omega$ under different values of the injection current $J$ for $C_{\mathrm{e}}=8$ and $\beta=3$.

characteristics. Figure 4 shows the relationship between the critical injection powers $\left(\varepsilon_{\mathrm{f}}, \varepsilon_{\mathrm{b}}\right)$ and the frequency detuning $(\Delta \Omega)$ under different bias currents for $C_{\mathrm{e}}=8$ and $\beta=3$, where Figs. $4 \mathbf{a}$ and $4 \mathbf{b}$ correspond to the case of increasing and decreasing $\varepsilon$, respectively. For forward scanning $\varepsilon$ as shown in Fig. $4 \mathbf{a}$, with the increase of $\Delta \Omega$, the injection power $\left(\varepsilon_{\mathrm{f}}\right)$ required for the state switching decreases gradually and exhibits relatively slight fluctuations for all current situations. For a given $\Delta \Omega, \varepsilon_{\mathrm{f}}$ gradually increases with the increase of the bias current. As for backward scanning $\varepsilon$ as shown in Fig. $4 \mathbf{b}$, the variation trend of $\varepsilon_{\mathrm{b}}$ with $\Delta \Omega$ is similar to $\varepsilon_{\mathrm{f}}$ in Fig. $4 \mathbf{a}$, but the fluctuation amplitude of $\varepsilon_{\mathrm{b}}$ gradually increases with the increase of the current. In order to reveal the underlying physical mechanism that $\varepsilon_{\mathrm{f}}$ and $\varepsilon_{\mathrm{b}}$ fluctuate with $\Delta \Omega$, Fig. 5 shows the

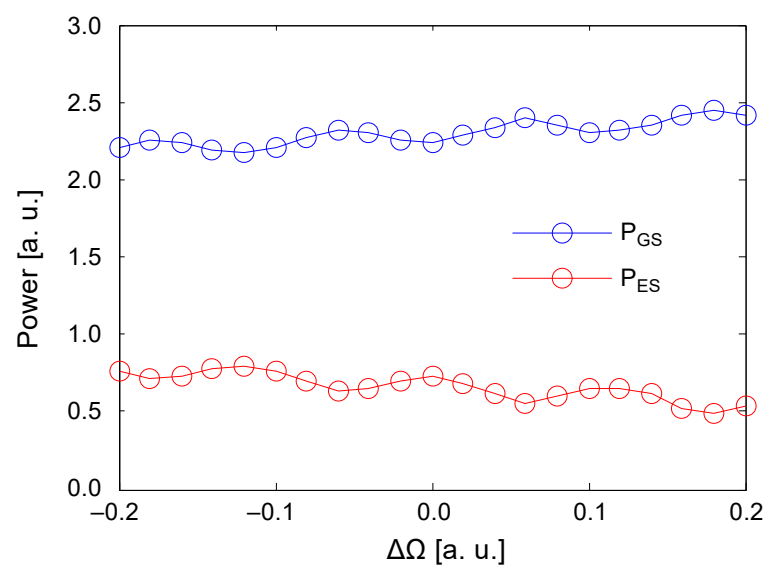

Fig. 5. GS and ES output powers $P_{\mathrm{GS}}$ and $P_{\mathrm{ES}}$ at the switching point versus the frequency detuning $\Delta \Omega$ for forward scanning under $J=6, C_{\mathrm{e}}=8$ and $\beta=3$. 


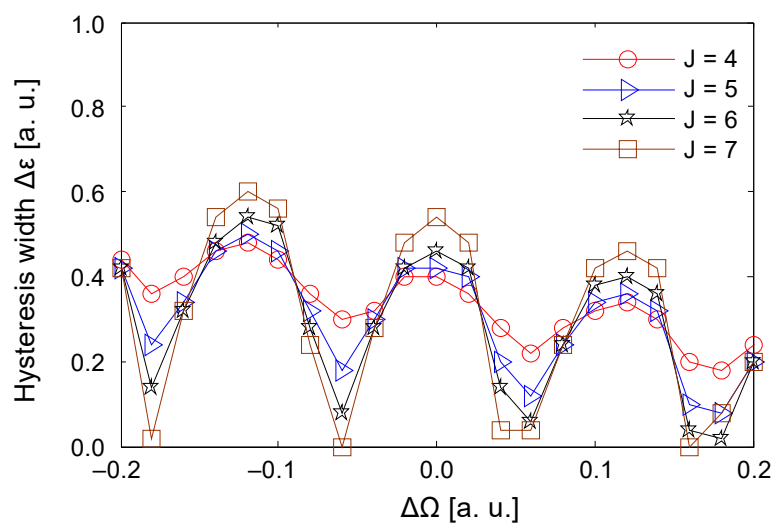

Fig. 6. Variations of the hysteresis width $\Delta \varepsilon$ with the frequency detuning $\Delta \Omega$ under different injection currents for $C_{\mathrm{e}}=8$ and $\beta=3$.

GS and ES switching output powers $\left(P_{\mathrm{GS}}, P_{\mathrm{ES}}\right)$ as a function of $\Delta \Omega$ for $J=6, C_{\mathrm{e}}=8$ and $\beta=3$ under the case of forward scanning. Due to special energy level structure of the two-state QD lasers, the introduction of an external perturbation such as optical feedback and optical injection may cause mode competition between GS and ES [31, 34, 39]. As can be seen in this diagram, with the increase of $\Delta \Omega, P_{\mathrm{ES}}$ and $P_{\mathrm{GS}}$ show an antiphase oscillation, where the variation trend of $P_{\mathrm{ES}}$ with $\Delta \Omega$ is similar to that of $\varepsilon_{\mathrm{f}}$ in Fig. 4a for $J=6$. As a result, we infer that the fluctuations of $\varepsilon_{\mathrm{f}}$ and $\varepsilon_{\mathrm{b}}$ with $\Delta \Omega$ may be due to the mode competition between GS and ES.

Since the hysteresis width is a key indicator in bistability applications, it is necessary to investigate the influence of the frequency detuning $\Delta \Omega$ and the injection current $J$ on the hysteresis width $\Delta \varepsilon$. Figure 6 presents the variation of $\Delta \varepsilon$ with $\Delta \Omega$ under different $J$. As shown in diagram, for a relatively small injection current $(J=4)$, with the increase of $\Delta \Omega$, the hysteresis width $\Delta \varepsilon$ decreases gradually and shows a slight fluctuation. With the increase of current, the fluctuation amplitude of $\Delta \varepsilon$ gradually increases. For $J=7$, the hysteresis disappears at two special frequency detuning points $(\Delta \Omega=-0.06$ and $0.16)$, which means that the critical injection powers $\left(\varepsilon_{\mathrm{f}}, \varepsilon_{\mathrm{b}}\right)$ for forward scanning and backward scanning are approximately equal (as shown in Fig. 4). For a relatively large current level, $\Delta \varepsilon$ is sensitive to the frequency detuning.

In the model used in this work, the inhomogeneous broadening factor $\beta$ is introduced to describe the phase-amplitude coupling between the ES and the GS [35]. In the rate equation of the two-state QD laser, only one QD ensemble is considered. However, the size distribution of QDs is not uniform during the growth process of QDs, which will lead to the change of carrier density [40]. The change of carrier density will cause the change of the refractive index and then results in the inhomogeneous broadening $[\underline{31}, \underline{36}]$. After introducing $\beta$, the theoretical simulation results agree well with the experimental results $[\underline{31}, \underline{36}]$. Figure 7 shows the relationship between the hysteresis loop widths and the inhomogeneous broadening factor $\beta$. For $\beta=3.5$ (Fig. 7a), 

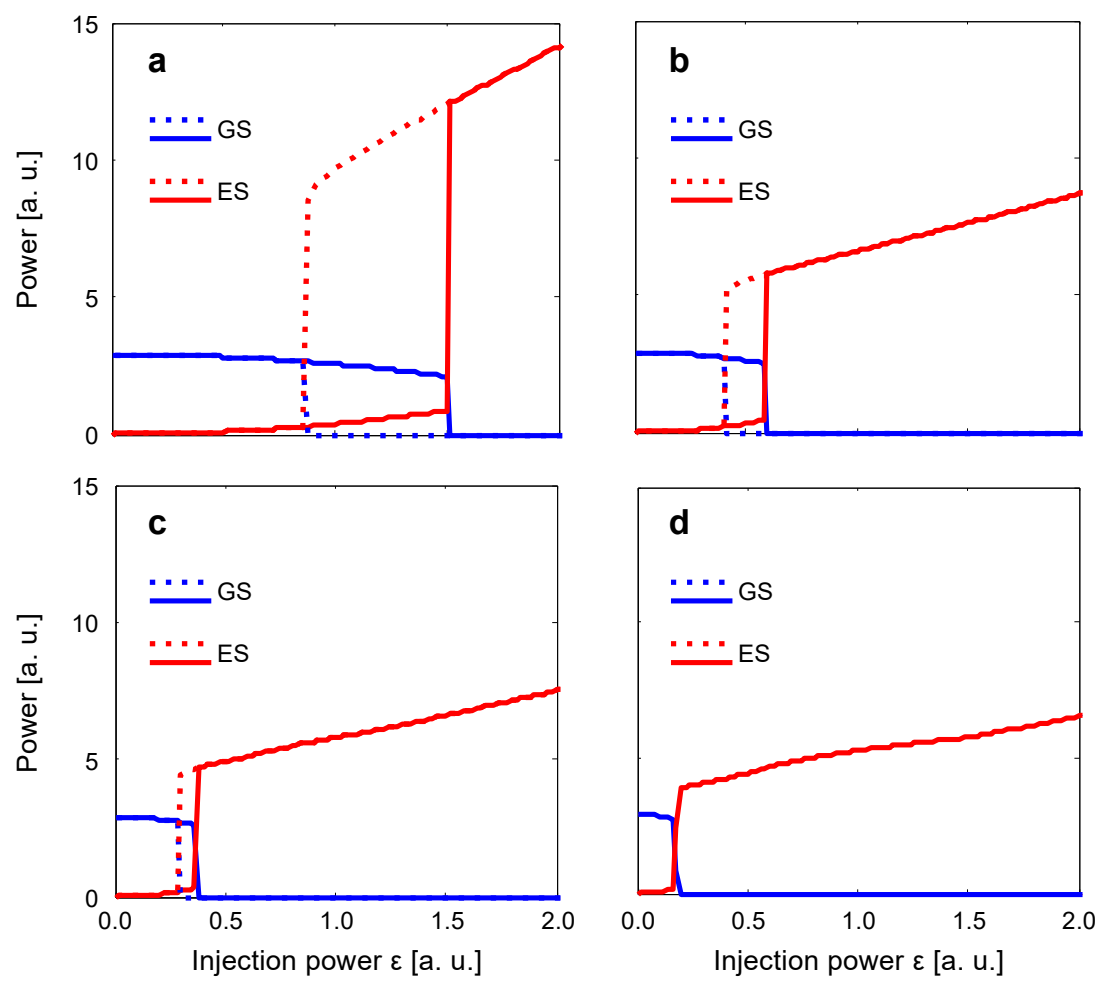

Fig. 7. Effect of the inhomogeneous broadening factor $\beta$ on the hysteresis width $\Delta \varepsilon$ under $J=6, \Delta \Omega=0$, and $C_{\mathrm{e}}=8$, where $\beta=3.5(\mathbf{a}), \beta=2(\mathbf{b}), \beta=1.5(\mathbf{c})$, and $\beta=1$ (d).

two clear hysteresis loops are obtained by scanning the injection power forward and backward, and the critical injection powers for the state switching are $\varepsilon_{\mathrm{f}}=1.50$ and $\varepsilon_{\mathrm{b}}=0.86$, and the corresponding hysteresis width $\Delta \varepsilon=0.64$. For $\beta=2$ (Fig. 7b), the critical injection powers are $\varepsilon_{\mathrm{f}}=0.58$ and $\varepsilon_{\mathrm{b}}=0.40$, the hysteresis width $\Delta \varepsilon=0.18$, and $\varepsilon_{\mathrm{f}}, \varepsilon_{\mathrm{b}}$ and $\Delta \varepsilon$ are reduced compared with those in Fig. 7a. For $\beta=1.5$ (Fig. 7c), the critical injection powers are $\varepsilon_{\mathrm{f}}=0.36$ and $\varepsilon_{\mathrm{b}}=0.28$, and $\Delta \varepsilon$ is further reduced to 0.08 . For $\beta=1$ (Fig. 7d), the critical injection powers $\varepsilon_{\mathrm{f}}$ and $\varepsilon_{\mathrm{b}}$ are equal, and the hysteresis disappears. From the above analysis, it can be seen that with the decrease of $\beta$, the hysteresis width decreases gradually until the hysteresis disappears, and the inhomogeneous broadening factor $\beta$ plays an important role in the bistability.

The above results are all based on the case of the electron escape rate $C_{\mathrm{e}}=8$. To show the influence of $C_{\mathrm{e}}$ on the bistability, Fig. 8 displays the variation of the hysteresis width $\Delta \varepsilon$ with $C_{\mathrm{e}}$ under different injection currents, where the values of $C_{\mathrm{e}}$ and $J$ in the figure guarantee that the free-running two-state QD laser is operating at the GS lasing. As observed from this diagram, for $C_{\mathrm{e}}<2$, all the hysteresis loop widths are almost 0 under various current conditions. For $C_{\mathrm{e}} \geq 2$, with the increase of $C_{\mathrm{e}}, \Delta \varepsilon$ increases gradually and shows weak fluctuations under different currents. Besides, with the increase 


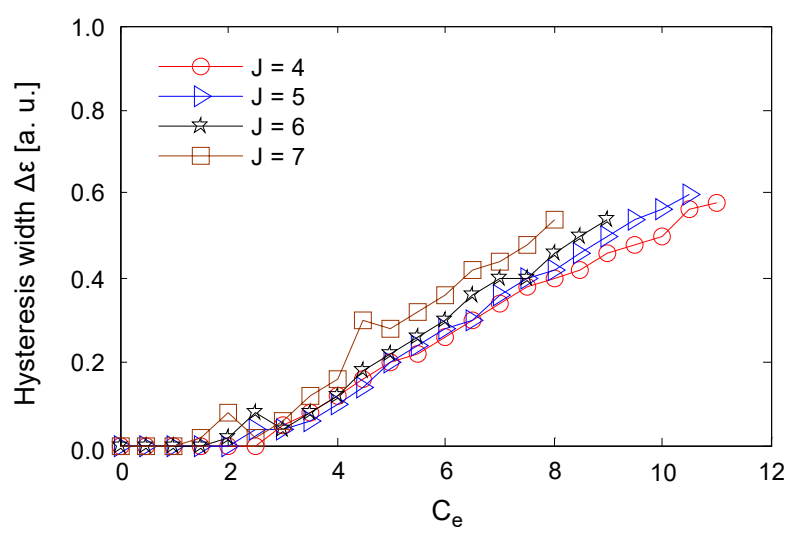

Fig. 8. Relationship between the hysteresis width $\Delta \varepsilon$ and the electron escape rate $C_{\mathrm{e}}$ under different injection current for $\Delta \Omega=0$ and $\beta=3$.

of the bias current, for a given $C_{\mathrm{e}}, \Delta \varepsilon$ gradually increases. As a result, the value of $C_{\mathrm{e}}$ will also affect the bistability.

\section{Conclusions}

In summary, the lasing state switching and bistability characteristics of a two-state QD laser subject to optical injection are investigated by using the electron-hole asymmetry model. For a free-running two-state QD laser operating at the GS under low bias current, the lasing state switching of the laser between the GS and the ES is dependent on the scanning paths of the injected light power when the frequency of the injection light is close to the ES lasing frequency. The bistability can be realized for the injection power within a certain region. The critical injection powers $\left(\varepsilon_{\mathrm{f}}, \varepsilon_{\mathrm{b}}\right)$ of the state switching decrease with increasing frequency detuning $\Delta \Omega$ and exhibit fluctuations. The width of the hysteresis loop fluctuates with $\Delta \Omega$, and the amplitude of the fluctuation increases with the increase of the injection current. In addition, the hysteresis disappears in two special frequency detuning points $(\Delta \Omega=-0.06$ and 0.16 ) for the injection current $J=7$. Also, the inhomogeneous broadening factor $\beta$ and the electron escape rate $C_{\mathrm{e}}$ have significantly influenced the bistability, with the decrease of $\beta$ and $C_{\mathrm{e}}$, the hysteresis width decreases gradually until the hysteresis disappears. Through adjusting the bias current or the frequency detuning, the characteristics of the lasing state switching and bistability in the two-state QD lasers subject to optical injection can be controlled to a certain extent. We believe that this work would be helpful for understanding the lasing state switching and bistability of the two-state QD lasers under optical injection and then exploiting related applications.

Acknowledgements - This work was supported by the National Nature Science Foundation of China (NSFC) under grant Nos. 61575163, 61775184 and 61875167. 


\section{References}

[1] Hill M.T., Dorren H.J.S., de Vries T., Leijtens X.J.M., den Besten J.H., Smalbrugge B., Oei Y., Binsma H., Kное G., Sмiт M.K., A fast low-power optical memory based on coupled micro-ring lasers, Nature 432, 2004, pp. 206-209, DOI: 10.1038/nature03045.

[2] Zhukovsky S.V., Chigrin D.N., Optical memory based on ultrafast wavelength switching in a bistable microlaser, Optics Letters 34(21), 2009, pp. 3310-3312, DOI: 10.1364/OL.34.003310.

[3] Salvide M.F., Masoller C., Torre M.S., All-optical stochastic logic gate based on a VCSEL with tunable optical injection, IEEE Journal of Quantum Electronics 49(10), 2013, pp. 886-893, DOI: 10.1109/JQE.2013.2276122.

[4] Takenaka M., Takeda K., Kanema Y., Nakano Y., Raburn M., Miyahara T., All-optical switching of $40 \mathrm{~Gb} /$ s packets by MMI-BLD optical label memory, Optics Express 14(22), 2006, pp. 10785-10789, DOI: $10.1364 / \mathrm{OE} .14 .010785$.

[5] Zhong D., Ji Y., Luo W., Controllable optoelectric composite logic gates based on the polarization switching in an optically injected VCSEL, Optics Express 23(23), 2015, pp. 29823-29833, DOI: 10.1364/OE.23.029823.

[6] Huybrechts K., Morthier G., Baets R., Fast all-optical flip-flop based on a single distributed feedback laser diode, Optics Express 16(15), 2008, pp. 11405-11410, DOI: 10.1364/OE.16.011405.

[7] Mori T., Sato Y. Kawaguchi H., Timing jitter reduction by all-optical signal regeneration using a polarization bistable VCSEL, Journal of Lightwave Technology 26(16), 2008, pp. 2946-2953.

[8] Brandonisio N., Heinricht P., Osborne S., Amann A., O’Brien S., Wavelength switching performance of single- and dual-contact two-mode semiconductor lasers with current modulation, Journal of Optics 13(12) 2011, article 125501, DOI: 10.1088/2040-8978/13/12/125501.

[9] Li S.-S., Li X.-Z., Zhuang J.-P., Mezosi G., Sorel M., Chan S.-C., Square-wave oscillations in a semiconductor ring laser subject to counter-directional delayed mutual feedback, Optics Letters 41(4), 2016, pp. 812-815, DOI: 10.1364/OL.41.000812.

[10] Blin S., Vaudel O., Besnard P., Gabet R., Power- or frequency-driven hysteresis for continuous -wave optically injected distributed-feedback semiconductor lasers, Optics Express 17(11), 2009, pp. 9288-9299, DOI: 10.1364/OE.17.009288.

[11] Zhang W.L., Pan W., Luo B., Wang M.Y., Zou X.H., Polarization switching and hysteresis of VCSELs with time-varying optical injection, IEEE Journal of Selected Topics in Quantum Electronics 14(3), 2008, pp. 889-894, DOI: 10.1109/JSTQE.2008.922896.

[12] Hurtado A., Quirce A., Valle A., Pesquera L., Adams M.J., Power and wavelength polarization bistability with very wide hysteresis cycles in a $1550 \mathrm{~nm}$-VCSEL subject to orthogonal optical injection, Optics Express 17(26), 2009, pp. 23637-23642, DOI: 10.1364/OE.17.023637.

[13] Chen J.-J., Wu Z.-M., Fan L., Tang X., Lin X.-D., Deng T., Xia G.-Q., Polarization bistability in a $1550 \mathrm{~nm}$ vertical-cavity surface-emitting laser subject to variable polarization optical injection, IEEE Photonics Journal 9(2), 2017, article 1502309, DOI: 10.1109/JPHOT.2017.2690661.

[14] Hong Y., Ju R., SPENCER P.S., SHORE K.A., Investigation of polarization bistability in vertical-cavity surface-emitting lasers subjected to optical feedback, IEEE Journal of Quantum Electronics 41(5), 2005, pp. 619-624, DOI: 10.1109/JQE.2005.845352.

[15] Tan S., Sun M., Lu D., Zhang R., Wang W., Ji C., Bistable 1060-nm high-power single-mode DFB laser diode, IEEE Photonics Journal 7(5), 2015, article 1503607, DOI: 10.1109/JPHOT.2015.2483203.

[16] Chen S., Li W., Wu J., Jiang Q., Tang M., Shutts S., Elliott S. N., Sobiesierski A., Seeds A.J., Ross I., SMowton P.M., Liu H., Electrically pumped continuous-wave III-V quantum dot lasers on silicon, Nature Photonics 10, 2016, pp. 307-311, DOI: 10.1038/nphoton.2016.21.

[17] Liu A.Y., Zhang C., Norman J., Snyder A., Lubyshev D., Fastenau J.M., Liu A.W.K., Gossard A.C., Bowers J.E., High performance continuous wave $1.3 \mu \mathrm{m}$ quantum dot lasers on silicon, Applied Physics Letters 104(4), 2014, article 041104, DOI: 10.1063/1.4863223. 
[18] Wang Y., Chen S., Yu Y., Zhou L., Liu L., Yang C., Liao M., Tang M., Liu Z., Wu J., Li W., Ross I., SeEds A.J., Liu H., Yu S., Monolithic quantum-dot distributed feedback laser array on silicon, Optica 5(5), 2018, pp. 528-533, DOI: 10.1364/OPTICA.5.000528.

[19] Jung D., Zhang Z., Norman J., Herrick R., Kennedy M.J., Patel P., Turnlund K., Jan C., Wan Y., Gossard A.C., Bowers J.E., Highly reliable low-threshold InAs quantum dot lasers on on-axis (001) Si with 87\% injection efficiency, ACS Photonics 5(3), 2018, pp. 1094-1100, DOI: 10.1021/ acsphotonics.7b01387.

[20] Ghalib B.A., Al-Obaidi S.J., Al-Khursan A.H., Modeling of synchronization in quantum dot semiconductor lasers, Optics and Laser Technology 48, 2013, pp. 453-460, DOI: 10.1016/j.optlastec. 2012.11.021.

[21] Liu H., Wang T., Jiang Q., Hogg R., Tutu F., Pozzi F., Seeds A., Long-wavelength InAs/GaAs quantum-dot laser diode monolithically grown on Ge substrate, Nature Photonics 5, 2011, pp. 416-419, DOI: $10.1038 /$ nphoton.2011.120.

[22] Li Q., Wang X., Zhang Z., Chen H., Huang Y., Hou C., Wang J., Zhang R., Ning J., Min J., ZHENG C., Development of modulation p-doped $1310 \mathrm{~nm}$ InAs/GaAs quantum dot laser materials and ultrashort cavity Fabry-Perot and distributed-feedback laser diodes, ACS Photonics 5(3), 2018, pp. 1084-1093, DOI: 10.1021/acsphotonics.7b01355.

[23] Liu H.Y., Childs D.T., Badcock T.J., Groom K.M., Sellers I.R., Hopkinson M., Hogg R.A., Robbins D.J., Mowbray D.J., Skolnick M.S., High-performance three-layer 1.3- $\mu$ m InAs-GaAs quantum-dot lasers with very low continuous-wave room-temperature threshold currents, IEEE Photonics Technology Letters 17(6), 2005, pp. 1139-1141, DOI: 10.1109/LPT.2005.846948.

[24] Sichkovskyi V.I., WANiczeK M., Reithmaier J.P., High-gain wavelength-stabilized $1.55 \mu \mathrm{m}$ InAs/ InP(100) based lasers with reduced number of quantum dot active layers, Applied Physics Letters 102(22), 2013, article 221117, DOI: 10.1063/1.4809730.

[25] Gao F., Luo S., Ji H.-M., YANG X.-G., YANG T., Enhanced performance of tunable external-cavity 1.5 m InAs/InP quantum dot lasers using facet coating, Applied Optics 54(3), 2015, pp. 472-476, DOI: $10.1364 / \mathrm{AO} .54 .000472$.

[26] Capua A., Rozenfeld L., Mikhelashyili V., Eisenstein G., Kuntz M., Laemmlin M., Bimberg D., Direct correlation between a highly damped modulation response and ultra low relative intensity noise in an InAs/GaAs quantum dot laser, Optics Express 15(9), 2007, pp. 5388-5393, DOI: 10.1364/ OE.15.005388.

[27] ShcheKin O.B., Deppe D.G., $1.3 \mu \mathrm{m}$ InAs quantum dot laser with $T_{0}=161 \mathrm{~K}$ from 0 to $80^{\circ} \mathrm{C}$, Applied Physics Letters 80(18), 2002, pp. 3277-3279, DOI: 10.1063/1.1476708.

[28] Sellin R.L., Ribbat C., Grundmann M., Ledentsov N.N., Bimberg D., Close-to-ideal device characteristics of high-power InGaAs/GaAs quantum dot lasers, Applied Physics Letters 78(9), 2001, pp. 1207-1209, DOI: 10.1063/1.1350596.

[29] Liu A.Y., Komljenovic T., Davenport M.L., Gossard A.C., Bowers J.E., Reflection sensitivity of $1.3 \mu \mathrm{m}$ quantum dot lasers epitaxially grown on silicon, Optics Express 25(9), 2017, pp. 9535-9543, DOI: $10.1364 /$ OE.25.009535.

[30] Abusaa M., Danckaert J., Viktorov E.A., Erneux T., Intradot time scales strongly affect the relaxation dynamics in quantum dot lasers, Physical Review A 87(6), 2013, article 063827, DOI: 10.1103/PhysRevA.87.063827.

[31] Viktorov E.A., Dubinkin I., Fedorov N., Erneux T., Tykalewicz B., Hegarty S.P., Huyet G., Goulding D., Kelleher B., Injection-induced, tunable, all-optical gating in a two-state quantum dot laser, Optics Letters 41(15), 2016, pp. 3555-3558, DOI: 10.1364/OL.41.003555.

[32] Meinecke S., Lingnau B., Röhm A., LüDGe K., Stability of optically injected two-state quantum-dot lasers, Annalen der Physik (Berlin) 529(12), 2017, p. 1600279, DOI: 10.1002/andp.201600279.

[33] Virte M., Breuer S., Sciamanna M., Panajotov K., Switching between ground and excited states by optical feedback in a quantum dot laser diode, Applied Physics Letters 105(12), 2014, article 121109, DOI: $10.1063 / 1.4896576$. 
[34] Virte M., Panajotov K., Sciamanna M., Mode competition induced by optical feedback in two-color quantum dot lasers, IEEE Journal of Quantum Electronics 49(7), 2013, pp. 578-585, DOI: $10.1109 /$ JQE.2013.2260725.

[35] Tykalewicz B., Goulding D., Hegarty S. P., Huyet G., Byrne D., Phelan R., Kelleher B., All-optical switching with a dual-state, single-section quantum dot laser via optical injection, Optics Letters 39(15), 2014, pp. 4607-4609, DOI: 10.1364/OL.39.004607.

[36] Tykalewicz B., Goulding D., Hegarty S.P., Huyet G., Dubinkin I., Fedorov N., Erneux T., Viktorov E.A., Kelleher B., Optically induced hysteresis in a two-state quantum dot laser, Optics Letters 41(5), 2016, pp. 1034-1037, DOI: 10.1364/OL.41.001034.

[37] Viktorov E.A., Mandel P., Electron-hole asymmetry and two-state lasing in quantum dot lasers, Applied Physics Letters 87(5), 2005, article 053113, DOI: 10.1063/1.1995947.

[38] Wang C., Zhuang J.-P., Grillot F., Chan S.-C., Contribution of off-resonant states to the phase noise of quantum dot lasers, Optics Express 24(26), 2016, pp. 29872-29880, DOI: 10.1364/OE.24.029872.

[39] Viktorov E. A., Mandel P., O’Driscoll I., Carroll O., Huyet G., Houlihan J., Tanguy Y., Low -frequency fluctuations in two-state quantum dot lasers, Optics Letters 31(15), 2006, pp. 2302-2304, DOI: $10.1364 /$ OL.31.002302.

[40] Gionnnini M., Montrosset I., Numerical analysis of the frequency chirp in quantum-dot semiconductor lasers, IEEE Journal of Quantum Electronics 43(10), 2007, pp. 941-949, DOI: 10.1109 / JQE.2007.904306. 